\title{
MOLECULAR DYNAMIC CALCULATION OF LATTICE THERMAL CONDUCTIVITY OF GOLD IN THE MELTING-CRYSTALLIZATION REGION
}

\author{
M.M. DEMIN, V.I. MAZHUKIN, A.A. ALEKSASHKINA
}

\author{
Keldysh Institute of Applied Mathematics of RAS, Russia, Moscow. \\ *Corresponding author. E-mail: vim@ modhef.ru
}

DOI: $10.20948 /$ mathmontis-2019-46-9

Summary. Of all the metals, gold is the most well-known and widely used material in scientific research, industrial production and, more recently, in biomedicine problems. In the temperature range $300 \leq \mathrm{T} \leq 2000 \mathrm{~K}$, including the region of the melting - crystallization phase transition, the results of modeling the phonon thermal conductivity of gold are presented. Phonon thermal conductivity plays an important role in modeling the mechanisms of interaction of pulsed laser radiation with gold in the framework of the two-temperature continuum model. In the region of the phase transition, overheating-undercooling of the solid phase occurs, the substance changes its structure. These phenomena are associated with changes in the phonon subsystem of gold, therefore, for mathematical modeling of heatingcooling, it is necessary to know the characteristic of heat transfer as the thermal conductivity of the phonon subsystem of gold. Obtaining the temperature dependence of phonon thermal conductivity in such a wide temperature range from experiment is problematic. In this work, phonon thermal conductivity was obtained by the direct non-equilibrium method in the framework of molecular dynamics modeling using the EAM potential.

\section{INTRODUCTION}

Of all metals, gold is the most well-known and widely used material in scientific research [1], industrial production [2] and, more recently, in biomedicine problems [3,4]. Besides various applications, the specific features of gold are of great fundamental interest in connection with the rapid development of new nanoscale materials [5]. Gold nanoparticles have unique properties [6,7], which served as the basis for their possible use for theranostics in nanomedicine [8].

One of the promising directions in the generation of nanoparticles and nanoclusters is laser ablation of materials by ultrashort (femto-picosecond) pulses [9, 10]. Femto-picosecond ablation occurs under conditions of strong thermodynamic non-equilibrium, which is characterized, in particular, by the presence of two temperatures: electron $T_{e}$ for the degenerate gas of free electrons and phonon $T_{\mathrm{ph}}$ for the crystal lattice. In the problems of mathematical modeling, the presence of two temperatures requires separation and quantitative determination of all thermodynamic and thermophysical characteristics of the material under study. Of all the thermophysical characteristics of metals, the separation and quantitative determination of phonon thermal conductivity was carried out less frequently than others. This was because it was believed that free electrons make the dominant contribution to thermal transfer in metals, while the phonon contribution to the total thermal conductivity was

2010 Mathematics Subject Classification: $82 \mathrm{C} 26,74 \mathrm{~A} 15,74 \mathrm{~A} 25$.

Key words and Phrases: Molecular Dynamics Simulation, Phonon Thermal Conductivity, Melting crystallization phase transition. 
considered negligible. Moreover, in most cases, knowledge of only the general thermal conductivity of metals was required; therefore, it was not necessary to separate the electronic and phonon thermal conductivity. The interest in quantifying phonon thermal conductivity in metals was stimulated primarily by the need for a deeper understanding of the mechanisms of thermal transfer during nonequilibrium energy transfer in a number of applications, for example, $[10,11]$.

Significant progress in recent years in the development of numerical methods and computational algorithms allows us to determine the phonon thermal conductivity for the most metals with a sufficient degree of accuracy. Two most common families of methods can be distinguished: classical molecular dynamics (MD) [12,13] and quantum ab-initio methods $[14,15]$. In the MD methods, when modeling the properties and processes in metals, the empirical and semi-empirical potentials of the "embedded atom method" (EAM) [16, 17] were obtained using the methods that include ab-initio ones [18]. In EAM potentials, in addition to pair interactions, collective interaction is also taken into account. As a result, the potential energy of the metal is represented as the sum of two potentials: the embedding potential of the i-th atom, which depends on the effective electron density in the region of the center of the atom and the pair potential. The main disadvantages of EAM potentials are not taking into account the phonon-electron interaction and the large number of fitting parameters included in it (up to two dozen). The choice of shape and the selection of parameters in the EAM potential can be carried out using quantum mechanical methods. These methods, implemented in the form of Abinit, VASP, SIESTA, and other software codes, make it possible to calculate the forces acting on each metal particle in a wide temperature range. Not all EAM potentials used allow a good description of both the crystalline and liquid phases of a metal. Therefore, when choosing the potential for atomistic modeling, careful testing of the potential used is necessary.

The ab-initio methods have appeared recently. They are considered the most promising and can be applied to any material. The ab-initio method does not require specifying interparticle potential. Its advantage is the ability to calculate electronic wave functions and electronic spectra. However, the use of small models does not guarantee the receipt of thermodynamic, structural and thermophysical properties with a sufficient degree of accuracy. The increase in model size is associated with high computational costs. Until recently, this was the main limitation that restrained the development and application of the ab-initio method. With the advent of high-performance computing, this limitation is successfully overcome.

The use of ab-initio methods in calculating phonon thermal conductivity allows one to take into account the influence of both phonon-phonon (p-p) and phonon-electron (p-e) interactions, which can significantly increase the reliability of the results. However, the number of calculations of the phonon thermal conductivity of metals and, in particular, gold [19], [20], [21], is currently relatively small. As a rule, all calculations are limited to the solid phase in the temperature range $T \sim(300-1000) \mathrm{K}$. There are no systematic results of experimental-theoretical studies of the properties of liquid metals in a wide temperature range (from the beginning of melting to the critical region).

At the same time, the knowledge of the temperature dependences of thermal conductivity is relevant in many aspects of materials science, from the kinetics of fast phase transitions [22], [23] to nanomicro processing of metal samples [24], [25].

The aim of this work is to obtain a quantitative dependence of the phonon thermal conductivity of gold in the temperature range, including the melting region ( $\mathrm{T} \sim 300-2000 \mathrm{~K})$. 
The goal was achieved by mathematical modeling, in which the atomistic model was numerically solved by the molecular dynamics method using a computational algorithm based on the finite-difference Verlet scheme [26]. The determination of phonon thermal conductivity in the framework of classical molecular dynamics is a complex problem. To solve it, the two most common approaches are used: the direct nonequilibrium method, which relies on spatial gradients of the type "heat source - sink" [27, 28, 29] and the equilibrium Green-Kubo method [27, 28, 30], associated with the calculation of time integrals of the correlation functions that specify the correlation in the systems with random processes.

In this work, to determine the phonon thermal conductivity of a metal ( $\mathrm{Au})$, we chose the direct nonequilibrium method as a more obvious one and requiring less time.

\section{STATEMENT OF THE PROBLEM}

Direct non-equilibrium method of determination of the phonon thermal conductivity coefficient $\kappa_{\text {lat }}$ is based on the phenomenological relation of Fourier for the heat flux $W$ [31]:

$$
W=-\kappa_{l a t} \frac{\partial T}{\partial x}
$$

The computational algorithm is constructed according to a scheme close to the experimental measurements of the coefficient $\kappa_{\text {lat }}$ [29, 32]. First, with the help of external heating in the sample, a stationary spatial temperature profile is constructed using the heat source - sink scheme, which provides a stationary heat flux. The presence of a stationary heat flux allows using the phenomenological Fourier relation (1) to determine the coefficient $\kappa_{\text {lat }}$.

A specific feature of the atomistic representation of processes in a solid sample is the choice of the linear dimensions of the sample (modeling domain), which must satisfy the following requirements.

In the molecular-dynamic implementation of the direct method, it is necessary to monitor the 2 most critical factors.

It is important that a stationary spatial temperature profile is obtained between the heat source and the sink, which is equivalent to obtaining a constant heat flux, with minimal finite size effect on the phonon thermal conductivity. For this, the linear dimensions of the sample should far exceed the average mean free path of phonons. The fulfillment of this condition is hampered by the fact that the dimensions of the computational domain should be of the micrometer scale, which in the calculations corresponds to a large number of atoms, several hundred million [33]. Keeping the computational domain in the nanometer range and performing calculations with a relatively small number of atoms is usually achieved using the scaling procedure [27], [28]. Its essence is as follows. First, using nanoscale calculation regions (along the $\mathrm{x}$ direction) of various lengths $L_{n}$, a series of calculations of thermal conductivity is carried out for several fixed values of temperature $T$. Then, the reciprocal dependence of the thermal conductivity $1 / \kappa_{\text {lat }}$ is constructed with respect to the reciprocal of the length of the simulation region, $1 / L_{n}$, and the thermal conductivity is determined by extrapolating the data $1 / L_{n} \rightarrow 0$ [27]. This procedure is justified by the expression for thermal conductivity obtained from the kinetic theory [27, 29].

In the direct method, large $\left(10^{9}-10^{10} \mathrm{~K} / \mathrm{m}\right)$ temperature gradients are used to reduce the temperature fluctuations. Too large temperature gradients can cause large nonlinear response 
effects for which the Fourier relation (1) is not applicable. In these cases, it is necessary to control the supply of heat to the source region with a thermostat, in order to determine the allowable value of non-linear distortions.

The calculation of the phonon thermal conductivity of gold was carried out as part of the following computational experiment. A region in the form of a parallelepiped with sizes of 10 $\times 10 \times 40$ unit cells, corresponding to 16,000 particles, with periodic boundary conditions in all three directions was considered. For boundary conditions along the $\mathrm{x}$ axis, this means that a particle exiting through the upper boundary of the region is replaced by a particle having the same velocity but entering through the lower boundary and vice versa. Gold has a cubic facecentered lattice, the lattice constant is $0.406 \mathrm{~nm}$. The interatomic interaction potential of EAM was used as the interaction potential [18].

The region along the $x$ axis was divided into the number of intervals corresponding to the number of unit cells along this axis, which in turn corresponds to the number of particles. Heating was performed in the first interval of the computational domain, the sink interval was located in the middle of the domain. At each time step, a fixed amount of heat $\delta Q_{N}$ was introduced into the heating region, and the same amount was taken from the sink region. The heat flux $W$ was calculated as

$$
W=d Q /(S N d t) / 2,
$$

where $d Q=N \times d t \times \delta Q_{N}$ is the total released energy, where $\delta Q_{N}$ is the energy released during one timestep, $N$ is the number of steps, $d t$ is the timestep size, $S$ is the cross-sectional area. The timestep $d t$ was chosen to be $3 \mathrm{fs}$ at low temperature $300 \leq T \leq 1000 \mathrm{~K}, 2$ fs for $1000<T \leq T_{m}$, and $1 \mathrm{fs}$ for $T>T_{m}$. The division by 2 is used due to periodic boundary conditions, i.e. heat flow goes in two directions. Then, the resulting temperature gradient was calculated, and the Fourier law (1) was used to obtain thermal conductivity.

\section{MODELING RESULTS}

Fig. 1 (a), (b) show the time-averaged spatial temperature profiles used to calculate the thermal conductivity. The average temperature is $300 \mathrm{~K}$ (Fig. 1 (a)) and $2000 \mathrm{~K}$ (Fig. 1 (b)). In a small region $(\sim 2 \mathrm{~nm})$ in the immediate vicinity of the source, a very strongly nonlinear temperature profile is observed. The same strongly nonlinear temperature profile is also observed near the sink. In the intermediate region, the behavior of the temperature profile is close to a linear dependence. This interval between the heat source and the heat sink is indicated by dashed lines in the graphs (Fig. 1 (a), (b)). This is where the temperature gradients were measured.

To overcome the effects of finite size, the heat flux was determined by a series of calculations. Calculations were carried out for various lengths of the computational domain $L_{n}: 40,80,160,240$, and 320 unit cells with a constant cross section of $10 \times 10$ cells at the same temperature.

The heat flux (2) was determined from the temperature difference between the heating and heat sink areas, for which the instantaneous temperature difference was averaged over the entire calculation time after establishing the stationary distribution. To increase the accuracy of the calculations, the temperature difference was calculated not over the entire interval between the source and the sink, but in its central part with a length of 0.8 of the full length. 
To calculate the thermal conductivity from the Fourier law (1), the scaling procedure described above was used. For each temperature value, an inverse dependence of the thermal conductivity $1 / \kappa_{\text {lat }}$ was constructed with respect to the reciprocal of the length $L_{n}$ of the calculation region, $1 / L_{n}$, and the thermal conductivity was determined by extrapolating the data $1 / L_{n} \rightarrow 0$. In Fig. 2 (a), (b) the dependences of the reciprocal of the thermal conductivity on the reciprocal of the size of the region for two temperatures $300 \mathrm{~K}$ and $2000 \mathrm{~K}$ are shown. MD simulation results are shown by a black line with markers.

We consider the scaling procedure using the example of obtaining thermal conductivity for a temperature $T=300 \mathrm{~K}$ (Fig. 2 (a)).
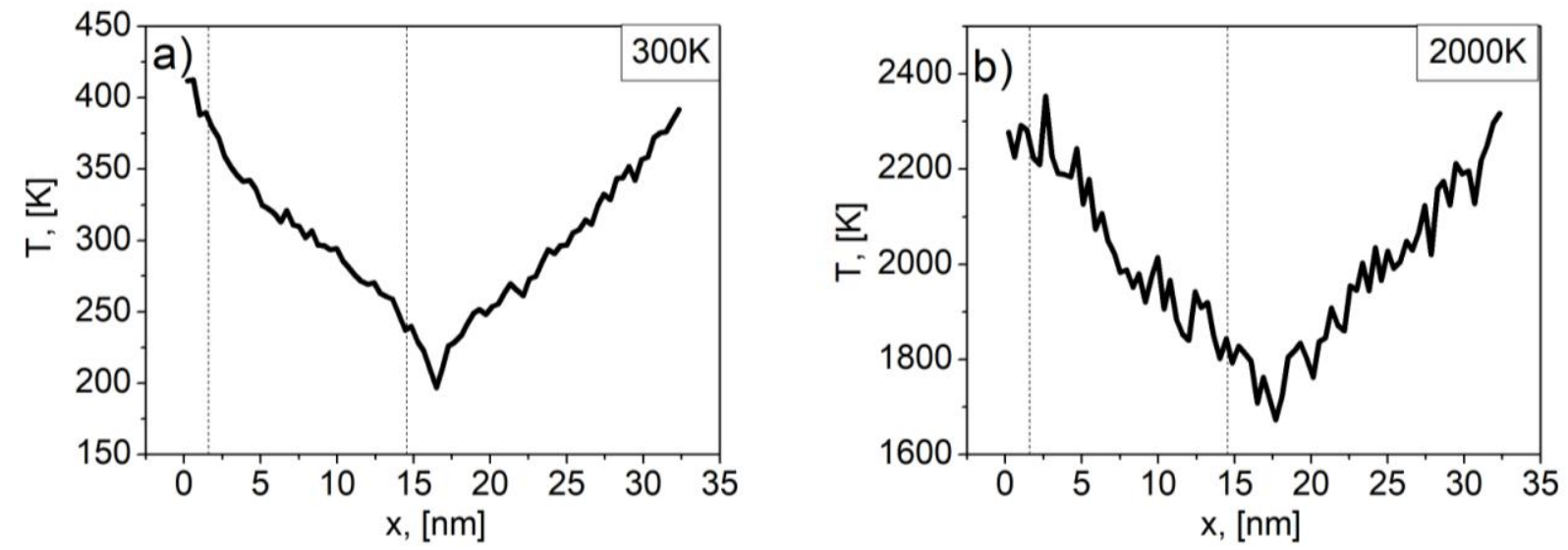

Fig.1 Spatial distribution of the temperature (a) at $300 \mathrm{~K}$ (b) at $2000 \mathrm{~K}$
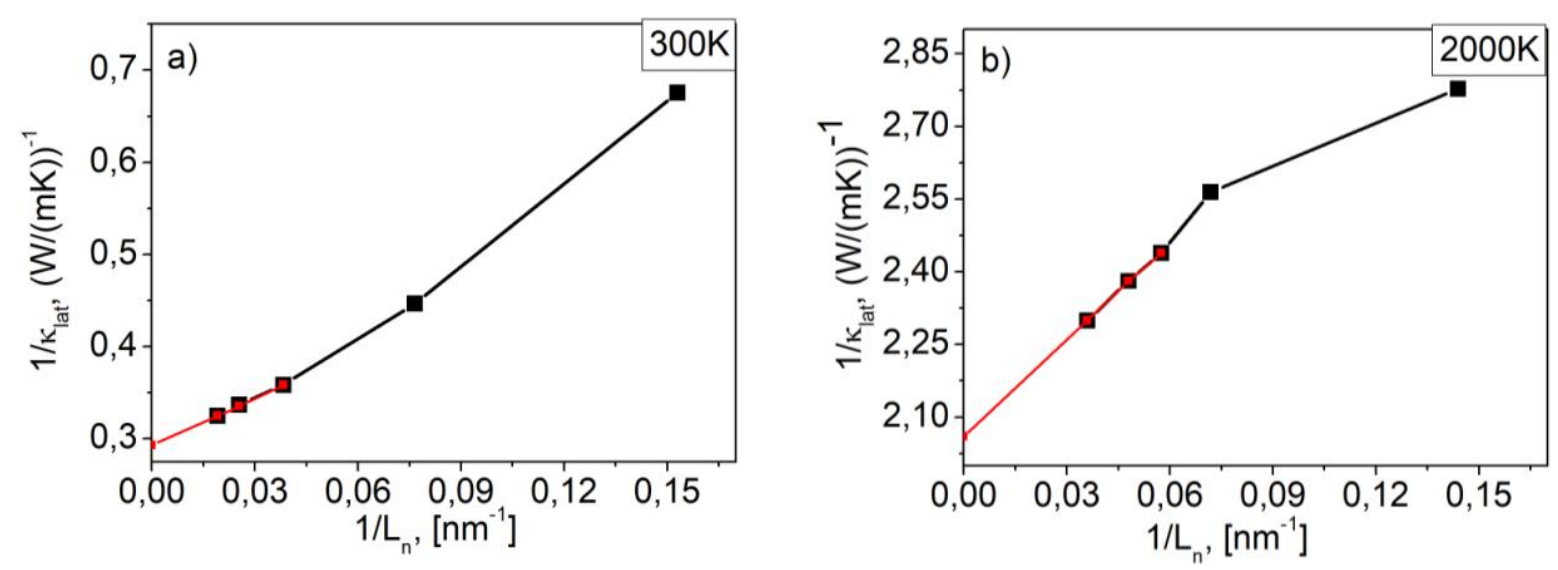

Fig.2. The dependence of the reciprocal of the thermal conductivity on the reciprocal of the length $\mathrm{L}_{\mathrm{n}}$ of the computational domain at temperatures: (a) $300 \mathrm{~K}$, (b) $2000 \mathrm{~K}$.

Using the least squares method, a linear dependence was obtained for three values of $1 / L_{n}$ ( $\mathrm{n}=1,2,3$ ), corresponding to the lengths of the computational domain $L_{1}=40, L_{2}=80$ and $L_{3}$ $=160$ cells $($ Fig. 2 (a)):

$$
P(z)=0.293+1.713 z
$$


In fig. 2 (a), the obtained dependence $P(z)$ is shown by a red line with markers. At $z=1 / L_{n}$ $=0$ we obtain the value corresponding to the infinite length of the computational domain. The reverse of this value is equal to the thermal conductivity at this temperature $(300 \mathrm{~K})$. The procedure was repeated for all necessary temperatures in the range $300 \mathrm{~K} \leq \mathrm{T} \leq 2000 \mathrm{~K}$.

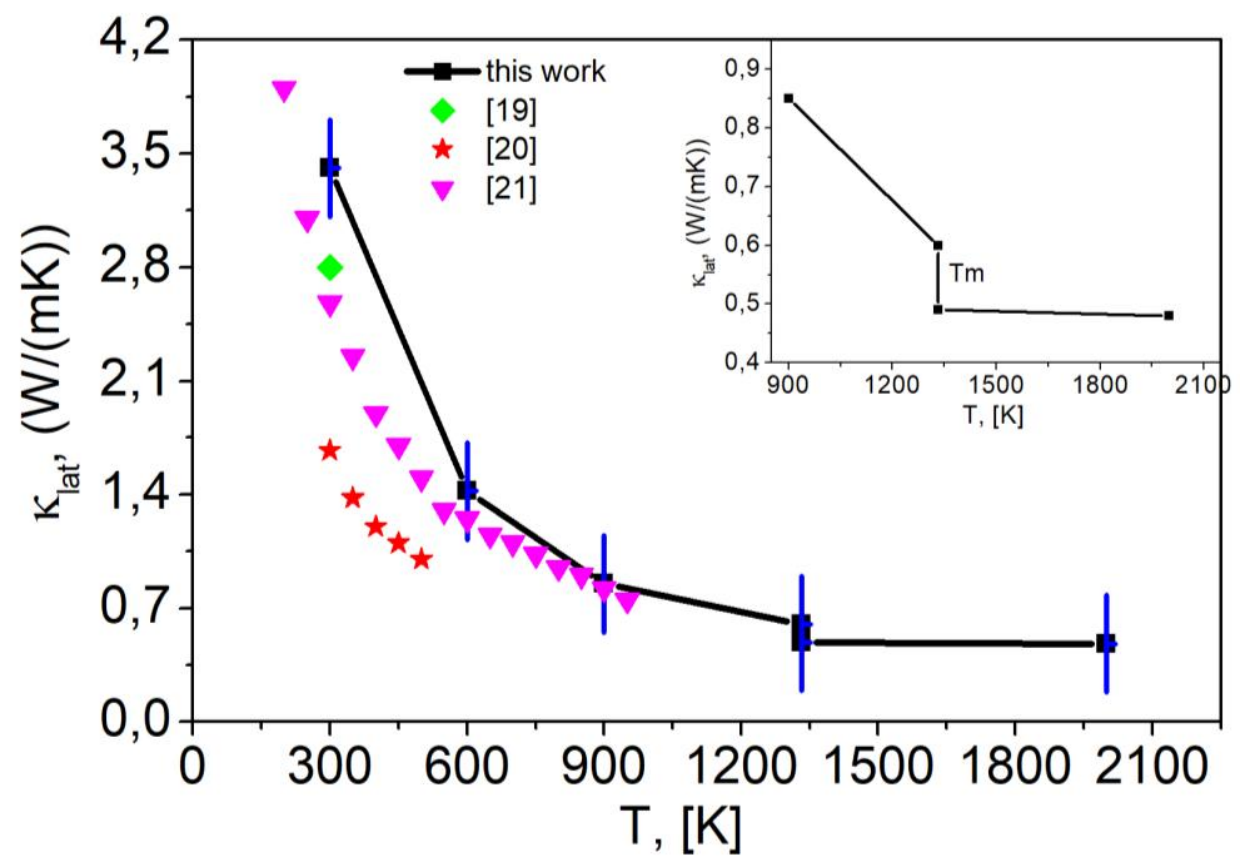

Fig.3. The temperature dependence of phonon themal conductivity of gold. The markers show the results of calculations by other authors.

Fig. 3 shows the temperature dependence of the phonon thermal conductivity of gold obtained as a result of the calculations. At the temperature of $300 \mathrm{~K}$, the phonon thermal conductivity is $\kappa_{\text {lat }}=3.41 \mathrm{~W} / \mathrm{mK}$. With increasing temperature, the thermal conductivity of gold decreases. At the equilibrium melting temperature $\mathrm{Tm}=1332 \mathrm{~K}$, the thermal conductivity in the solid phase is $\kappa_{\text {lat }}=0.6 \mathrm{~W} / \mathrm{mK}$, and in the liquid phase at the same temperature the thermal conductivity is $\kappa_{\text {lat }}=0.49 \mathrm{~W} / \mathrm{mK}$. Thus, an abrupt decrease in thermal conductivity by $0.11 \mathrm{~W} / \mathrm{mK}$ was obtained (inset in Fig. 3), which is $18 \%$. The calculation was carried out up to a temperature $\mathrm{T}=2000 \mathrm{~K}$, at which the thermal conductivity is $\kappa_{\text {lat }}=0.48 \mathrm{~W} / \mathrm{mK}$. Such a change in the phonon thermal conductivity with increasing temperature does not contradict the ideas about the behavior of the phonon thermal conductivity of metals.

The comparison with alternative calculations [19-21] showed a good agreement. At low temperatures $(300 \mathrm{~K} \leq T<600 \mathrm{~K})$, the largest difference with $[19,21]$ is $\Delta \kappa \sim 32 \%$. With increasing temperature, the difference in results becomes smaller. At $T=600 \mathrm{~K}$ [21], the difference is $\Delta \kappa \sim 12 \%$, and at $T=900 \mathrm{~K}$ almost completely coincides $\Delta \kappa \sim 2 \%$. The consistency with the results of [20] is lesser. In the temperature range $T>1000 \mathrm{~K}$, there is no data for comparison. In general, such comparison results suggest that the selected method and potential describe the model with good accuracy and are applicable for further studies.

The calculations were performed using the LAMMPS package [34] 


\section{CONCLUSIONS}

In the course of molecular dynamics experiments using the direct nonequilibrium method with the EAM potential [18], the phonon thermal conductivity of gold was calculated in the temperature range from $300 \mathrm{~K}$ to $2000 \mathrm{~K}$. The indicated interval includes a region of a firstorder phase transition (melting). At the melting point, the phonon thermal conductivity is calculated for two states of matter: solid and liquid.

A comparison of the obtained temperature dependence of the phonon thermal conductivity coefficient with the results from [19, 20,21] shows a good agreement.

Acknowledgements: This work was supported by Russian Science Foundation (project No. 18-11-00318).

\section{REFERENCES}

[1] G.A. Shafeev, I.I. Rakov, K.O. Ayyyzhy, G.N. Mikhailova, A.V. Troitskii, O.V. Uvarov, "Generation of $\mathrm{Au}$ nanorods by laser ablation in liquid and their further elongation in external magnetic field", Applied Surface Science, 466, 477-482 (2019). doi:10.1016/j.apsusc.2018.10.062

[2] Sandra Jendrzej, Bilal Gökce, Matthias Epple, Stephan Barcikowski, "How Size Determines the Value of Gold - Economic Aspects of Wet Chemical and Laser-Based Metal Colloid Synthesis", ChemPhysChem., 18, 1-9 (2017). DOI: 10.1002/cphc.201601139

[3] N. Elahi, M. Kamali, M. H. Baghersad, "Recent biomedical applications of gold nanoparticles: A review", Talanta, 184, 537-556, (2018) https://doi.org/10.1016/j.talanta.2018.02.088

[4] X. Zhang, "Gold Nanoparticles: Recent Advances in the Biomedical Applications", Springer, 72 (3), 771-775 (2015).

[5] S. Barcikowski, A. Hahn, A.V. Kabashin, B.N. Chichkov, "Properties of nanoparticles generated during femtosecond laser machiningin air and water", Appl. Phys. A, 87, 47-55 (2007). DOI: 10.1007/s00339-006-3852-1

[6] K. Maximova, A. Aristov, M. Sentis, A.V. Kabashin, "Size-controllable synthesis of bare gold nanoparticles by femtosecond laser fragmentation in water", Nanotechnology, 26, 065601 (8pp) (2015).

[7] Paulina Abrica-González, J. A. Zamora-Justo, Blanca Estela Chavez-Sandoval, José Abraham Balderas-López, "Measurement of the Optical Properties of Gold Colloids by Photoacoustic Spectroscopy", Int. J. Thermophysics, 39(8), 93 (2018) DOI: 10.1007/s10765-018-2412-1

[8] X. Xue, \& X.-J. Liang, "Multifunctional Nanoparticles for Theranostics and Imaging. Nanostructure Science and Technology", Y. Ge et al. (Eds.) in Nanomedicine, Nanostructure Science and Technology, Springer Science+Business Media New York, 101-115 (2014). doi:10.1007/978-1-4614-2140-5_6

[9] E.G. Gamaly, "The physics of ultra-short laser interaction with solids at non-relativistic intensities”, Physics Reports, 508(4-5), 91-243 (2011). doi:10.1016/j.physrep.2011.07.002

[10] A.V. Mazhukin, V.I. Mazhukin, M.M. Demin, "Modeling of femtosecond laser ablation of Al film by laser pulses", Applied Surface Science, 257, 5443-5446 (2011).

[11] R. Venkatasubramanian, E. Siivola, T. Colpitts and B. O. Quinn, "Thin Film Thermoelectric Devices with High Room Temperature Figures of Merit", Nature, 413, 597-602 (2001). http://dx.doi.org/10.1038/35098012

[12] F.F. Abraham, "Computational statistical mechanics: methodology, applications and supercomputing", Advances in Physics, 35, 1-111 (1986).

[13] A.F. Bakker, G.H. Gilmer, M.H. Grabow, and K. Thompson, "A special purpose computer for molecular dynamics calculations", J. Comp. Phys., 90, 313-335 (1990). 
[14] R. Car, M. Parrinello, "Unified approach for molecular dynamics and Density-Functional theory", Phys. Rev. Lett., 55, 2471 (1985).

[15] D. Marx, J. Hutter, Ab Initio Molecular Dynamics: Basic Theory and Advanced Methods, Cambridge University Press, New York (2009). ISBN 978-0521898638

[16] S.M. Foiles, M.I. Baskes, and M.S. Daw, "Embedded-atom-method functions for the fcc metals Cu, Ag, Au, Ni, Pd, Pt, and their alloys", Physical Review B, 33(12), 7983-7991 (1986). DOI: $10.1103 /$ physrevb.33.7983.

[17] S.V. Starikov, A.Y. Faenov, T.A. Pikuz, I.Y. Skobelev, V.E. Fortov, S. Tamotsu, M. Ishino, M. Tanaka, N. Hasegawa, M. Nishikino, T. Kaihori, T. Imazono, M. Kando, and T. Kawachi, "Soft picosecond X-ray laser nanomodification of gold and aluminum surfaces", Applied Physics $B$, 116(4), 1005-1016 (2014). DOI: 10.1007/s00340-014-5789-y.

[18] V.V. Zhakhovskii, N.A. Inogamov, Yu.V. Petrov, S.I. Ashitkov, K. Nishihara, "Molecular dynamics simulation of femtosecond ablation and spallation with different interatomic potentials", Appl. Surf. Sci., 255, 9592-9596 (2009)

[19] Z. Tong, S. Li, X. Ruan, and H. Bao, "Comprehensive first-principles analysis of phonon thermal conductivity and electron-phonon coupling in different metals", Physical review B, 100, 144306 (2019)

[20] A. Jain and A.J.H. McGaughey, "Thermal transport by phonons and electrons in aluminum, silver, and gold from first principles", Physical review B, 93, 081206(R) (2016).

[21] Y. Wang, Z. Lu, and X. Ruan, "First principles calculation of lattice thermal conductivity of metals considering phonon-phonon and phonon-electron scattering", Journal of applied physics, 119, 225109 (2016)

[22] V.I. Mazhukin, "Kinetics and Dynamics of Phase Transformations in Metals Under Action of Ultra-Short High-Power Laser Pulses", Chapter 8, I. Peshko (Ed.), in Laser Pulses - Theory, Technology, and Applications, InTech, Croatia, 219 -276 (2012).

[23] V.I. Mazhukin, A.V. Shapranov, A.V. Mazhukin, O.N. Koroleva, "Mathematical formulation of a kinetic version of Stefan problem for heterogeneous melting/crystallization of metals", Math. Montis., 36, 58-77 (2016).

[24] V.I. Mazhukin, A.V. Mazhukin, M.M. Demin, A.V. Shapranov, "Nanosecond laser ablation of target Al in a gaseous medium: explosive boiling", Appl. Phys. A, 124 (3), 237 (1-10) (2018). https://doi.org/10.1007/s00339-018-1663-9.

[25] V.I. Mazhukin, "Nanosecond Laser Ablation: Mathematical Models, Computational Algorithms, Modeling", Chapter 2, Tatiana E. Itina (Ed.) In Laser Ablation - From Fundamentals to Applications, InTech, Croatia, 31-55 (2017).

[26] L. Verlet, "Computer "Experiments" on Classical Fluids. I. Thermodynamically Properties of Lennard-Jones Molecules", Phys. Rev., 159, 98-103 (1967).

[27] P. K. Schelling, S. R. Phillpot, P. Keblinski, "Comparison of atomic-level simulation methods for computing thermal conductivity", Phys. Rev. B, 65, 144306 (2002)

[28] L. Hu, W. J. Evans, P. Keblinski, "One-dimensional phonon effects in direct molecular dynamics method for thermal conductivity determination", J. Appl. Phys., 110, 113511 (2011)

[29] Florian Müller-Plathe, "A simple nonequilibrium molecular dynamics method for calculating the thermal conductivity", J. Chem. Phys., 106, 6082 (1997).

[30] R. Zwanzig, "Time-correlation functions and transport coefficients in statistical mechanics", Annu. Rev. Phys. Chem., 16, 67-102 (1965).

[31] W. Evans and P. Keblinski, "Thermal conductivity of carbon nanotube cross-bar structures", Nanotechnology, 21(47), 475704, (2010) doi: 10.1088/0957-4484/21/47/475704

[32] Shenghong Ju and Xingang Liang, "Thermal conductivity of nanocrystalline silicon by direct molecular dynamics simulation”, J. Appl. Phys., 112, 064305 (2012) 
[33] Chaofeng Hou, Ji Xu, Wei Ge and Jinghai Li, "Molecular dynamics simulation overcoming the finite size effects of thermal conductivity of bulk silicon and silicon nanowires", Modelling Simul. Mater. Sci. Eng., 24, 045005 (1-9) (2016)

[34] S. Plimpton, "Fast parallel algorithms for short-range molecular dynamics", J. Comput. Phys., 117(1), 1-19 (1995).

Received August 15, 2019 